\title{
Effect of current density and etching time on photoluminescence and energy band gap of p-type porous silicon
}

\begin{abstract}
Porous silicon samples were formed on p-type silicon wafer by electrochemical method. Metal porous silicon structure was used to obtain the rectifying behavior. Current density and etching time was studied which affect the photoluminescence and energy band gap. Three different groups were prepared using different current density and varying etching time. The optical properties for p-type porous silicon were investigated by photoluminescence (PL) spectroscopy. Findings from study showed that the porous silicon has band gap energy in the range from 1.81 to $2.07 \mathrm{eV}$. The band gap energy also increased with increasing current density or increasing etching time. The PL peaks showed a steady red color shift from 500 to $800 \mathrm{~nm}$ with increasing etching time.
\end{abstract}

Keyword: Current densities; Energy band gap; Etching time; Porous silicon 